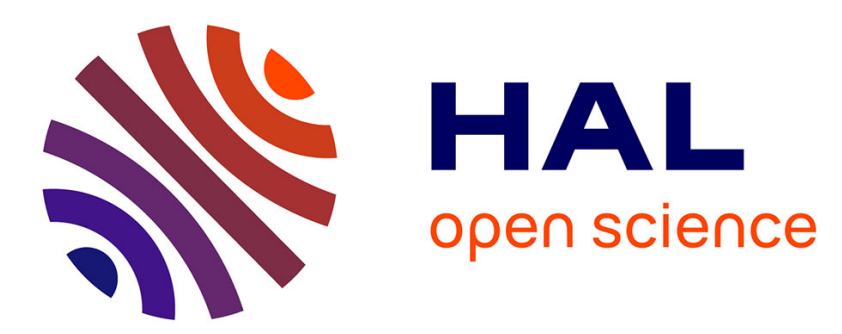

\title{
Mission design through averaging of perturbed Keplerian systems: the paradigm of an Enceladus orbiter
}

Martín Lara, Jesús F. Palacián, Ryan P. Russell

\section{To cite this version:}

Martín Lara, Jesús F. Palacián, Ryan P. Russell. Mission design through averaging of perturbed Keplerian systems: the paradigm of an Enceladus orbiter. Celestial Mechanics and Dynamical Astronomy, 2010, 108 (1), pp.1-22. 10.1007/s10569-010-9286-2 . hal-00568375

\section{HAL Id: hal-00568375 \\ https://hal.science/hal-00568375}

Submitted on 23 Feb 2011

HAL is a multi-disciplinary open access archive for the deposit and dissemination of scientific research documents, whether they are published or not. The documents may come from teaching and research institutions in France or abroad, or from public or private research centers.
L'archive ouverte pluridisciplinaire HAL, est destinée au dépôt et à la diffusion de documents scientifiques de niveau recherche, publiés ou non, émanant des établissements d'enseignement et de recherche français ou étrangers, des laboratoires publics ou privés. 


\title{
Mission Design Through Averaging of Perturbed Keplerian Systems: The Paradigm of an Enceladus Orbiter
}

\author{
Martín Lara, ${ }^{*}$ Jesús F. Palacián, ${ }^{\dagger}$ and Ryan P. Russell ${ }^{\ddagger}$
}

May 6, 2010

\begin{abstract}
Preliminary mission design for planetary satellite orbiters requires a deep knowledge of the long term dynamics that is typically obtained through averaging techniques. The problem is usually formulated in the Hamiltonian setting as a sum of the principal part, which is given through the Kepler problem, plus a small perturbation that depends on the specific features of the mission, but which is usually derived from a scaling procedure of the restricted three body problem, since the two main bodies are the Sun and the planet whereas the satellite is considered as a massless particle. Sometimes, instead of the restricted three body problem, the spatial Hill problem is used. In some cases the validity of the averaging is limited to prohibitively small regions, thus, depriving the analysis of significance. We find this paradigm at Enceladus, where the validity of a first order averaging based on the Hill problem lies inside the body. However, this fact does not invalidate the technique as perturbation methods are used to reach higher orders in the averaging process. Proceeding this way, we average the Hill problem up to the sixth order obtaining valuable information on the dynamics close to Enceladus. The averaging is performed through Lie transformations and two different transformations are applied. Firstly, the mean motion is normalized whereas the goal of the second transformation is to remove the appearance of the argument of the node. The resulting Hamiltonian defines a systems of one degree of freedom whose dynamics is analyzed.
\end{abstract}

\footnotetext{
${ }^{*}$ Ephemerides section, Real Observatorio de la Armada, ES-11 110 San Fernando, Spain

${ }^{\dagger}$ Dep. Ingeniería Matemática e Informática, Universidad Pública de Navarra, ES-31 006 Pamplona, Spain

${ }^{\ddagger}$ Guggenheim School of Aerospace Engineering, Georgia Institute of Technology, Atlanta, GA, 30 332-0150
} 


\section{Introduction}

The preliminary mission design of a science orbit around a planetary satellite requires a deep knowledge of the relevant dynamics. A careful analysis of the dominant, long term perturbations permits us to predict the orbital element changes that potentially drive the overall mission design.

The solution to many problems of interest consists of perturbed conics. In these cases the problem can be formulated as a perturbed two-body problem, and the Lagrange Planetary Equations of motion are suitable. If the perturbation is sufficiently small, then the long term dynamics are accessible through averaging after a removal of the short period terms.

When the perturbation of the Keplerian orbits is not small, however, the simple removal of short period effects from the disturbing function can fail in providing reliable information. The validity of the averaging may be limited to very small regions, thus, depriving the analysis of significance. In these cases, different alternatives may help in preliminary space mission design, which range from dynamical systems theory (see Refs. [37,51], for instance) to the determination of stability regions via the continuation of families of periodic orbits [34,31], the massive search and characterization of periodic orbits [43], or to carrying out direct numerical simulations [1], sometimes combined with differential corrections procedures [12]. However, if the trajectories still resemble Keplerian orbits, the fact that perturbations are not small does not completely invalidate the averaging technique, and higher orders in the averaging may be achieved with perturbation methods.

This paradigm is found in the study of close orbits about Enceladus: When averaging a perturbing function based on the Hill problem approach, it is found that the radius of validity of the averaging lies inside the body [ 47 , $44,45]$. But computing higher orders in the averaging of the Hill problem is a simple matter of iterating an algorithmic procedure $[20,9,6]$ —assumed, of course, that one can analytically solve the quadratures or partial differential equations that appear in the sequence - and it considerably extends the radius of validity of the averaging $[46,28] .{ }^{1}$ Proceeding this way, we reach a sixth order in the averaging of the Hill problem, and show that this higher order averaging provides valuable information on the dynamics of high altitude orbits up to at least half the Hill radius — about $500 \mathrm{~km}$ in the Saturn-Enceladus system, or two times Enceladus' equatorial radius.

A preliminary version of our study was presented in the 19th AAS/AAIA

\footnotetext{
${ }^{1}$ The resulting series are known to lack convergence, but it has no effect in the short intervals considered in Astrodynamics
} 
Spaceflight Mechanics meeting, held in 2009, see [29].

The paper is structured as follows. In Section 2 we present the problem and study the flow of the twice averaged problem, determining the classical periodic orbits and bifurcations. The goal of Section 3 is to apply the findings of Section 2 to the system Saturn-Enceladus. We show the benefists of the sixth order theory compared to lower order theories. In Section 4 we check the results of our analytical theory against a realistic (numerical) model ephemeris of an orbiter around Enceladus. In Section 5 the main conclusions of the paper are drawn. Finally an appendix is devoted to estimate the validity of our averaging procedure.

\section{Spatial Hill model dynamics}

\subsection{Hamiltonian of the problem and planar periodic orbits}

Since Hill's original contribution to the study of the motion of the Moon [19], Hill problem has been extensively studied. It provides a good approximation to the real dynamics of a variety of systems, encompassing the motion of comets, natural and artificial satellites, distant moons of asteroids, or dynamical astronomy applications $[35,17,14]$. Specifically, Hill model and its variations are useful for describing motion about planetary satellites $[24,36,47,33,42]$. In addition, Hill problem is an invariant model that does not depend on any parameter, thus, giving broad generality to the results, whose application to different systems becomes a simple matter of scaling.

Hill problem is a limit case of the Circular Restricted Three-Body Problem (CRTBP). A third body of negligible mass moves under the action of two primaries, which evolve in Keplerian circular orbits around each other. The minor primary is at the origin, and the reference frame is rotating with the mean motion of the primaries. We assume that the motion of the third body takes place close to the central body, its radius $r$ is small when compared with the distance between the primaries $R$, and the mass of the bigger primary is much larger than that of the central body. Under these assumptions, the equations of motion of the CRTBP may be developed in power series of $r / R$ and truncate to the first order. Therefore, the equations of motion of Hill problem are derived from the Hamiltonian:

$$
\mathcal{H}=\frac{1}{2}(\boldsymbol{X} \cdot \boldsymbol{X})-\boldsymbol{N} \cdot(\boldsymbol{x} \times \boldsymbol{X})+W(\boldsymbol{x}), \quad W=\frac{N^{2}}{2}\left(r^{2}-3 x^{2}\right)-\frac{\mu}{r},
$$

where $\boldsymbol{x}=(x, y, z)$ is the position vector, $\boldsymbol{X}=(X, Y, Z)$ is the vector of 
conjugate momenta, $r=\|\boldsymbol{x}\|$, and both the rotation rate of the system $N=\|\boldsymbol{N}\|$ and the gravitational parameter $\mu$ of the central body may be set to 1 in appropriate units. The problem is of three degrees of freedom, yet admitting the Jacobi constant $\mathcal{H}=-C / 2$.

Insight regarding stability in Hill model is available through the computation of families of periodic orbits in the rotating frame $[48,16,13]$, where information on the stability character of each periodic orbit is easily obtained $[15,2,18]$. Planar, retrograde periodic orbits are generally stable, and, on the contrary, corresponding planar, direct periodic orbits change to instability relatively close to the central body $[48,16,17,31]$. Figure 1 shows the stability curves of the family of planar, direct, periodic orbits. The family starts with almost circular orbits close to the origin, but after the change to (vertical) instability at $C=4.5$ (corresponding to an average distance of less than 0.3 in Hill units), they evolve to unstable ellipses centered at the origin in the rotating frame, which shape clearly departs from Keplerian ellipses in the inertial frame. Note that the planar retrograde orbits exist well beyond the Hill radius while the direct planar orbits are strictly constrained to the region inside Hill radius.

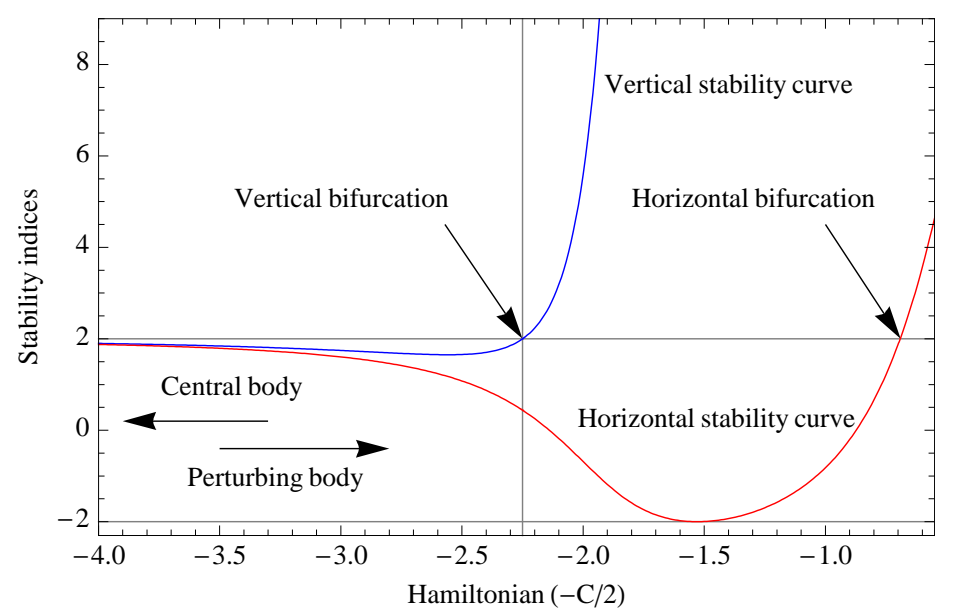

Figure 1: Stability diagram for direct, planar periodic orbits of Hill problem.

The continuation of three dimensional periodic orbits that bifurcate vertically from planar resonant orbits help in determining stability regions in 3-D [34, 32]. An important recent result shows that stability regions of direct inclination orbits extends to higher inclinations than in the retrograde case $[31,43]$.

Alternatively, Hill problem may be studied by averaging (see Ref. [3] and 
references therein). After removing the short and medium periodic terms from the perturbing function of the Hill problem, the Lagrange planetary equations capture the dominant characteristics of the dynamics.

The averaging is limited to regions where the ratio between the mean motions of the system $N$ and the orbiter $n$ is small [47,44,45]. Nevertheless, the use of perturbation methods may extend the applicability of the averaging to larger distances, and approximate solutions up to the fourth order in the ratio $N / n$ have been reached recently [28]. In this context we should mention previous work by the Russian school where theories of third order in the ratio $N / n$ have been obtained $[22,39,49,50]$. However, even the case of Enceladus the overwhelming presence of Saturn makes the dynamical environment highly unstable, and even the fourth order perturbation theory has a limited applicability.

Needless to say, typical analytical theories based on perturbation methods are limited to the case of perturbed Keplerian ellipses [6,40]. Therefore, the Jacobi constant (or Hamiltonian) value at which planar, direct, periodic orbits change their shape from almost circular to oval shaped, provides a conservative limit for the application of analytical theories of Hill problem describing motion around the central body. However, high inclination orbits are known to be less distorted, and one may hopefully expect that this conservative limit is to be enlarged and analytical results may apply further for high inclination orbits. Specifically, this applies to the region of stable, low-eccentricity, high-inclination, direct periodic orbits of Hill problem that exist up to about half the Hill radius from the origin [43,30]. After scaling Hill problem for the Saturn-Enceladus system parameters, it has been demonstrated that orbits in this region show long term stability in ephemeris models [45], and corresponding orbits have been proposed for a prospective science mission to Enceladus [5].

\subsection{Perturbation theory}

Close to the central body Hill problem can be studied as a perturbed two body problem. In usual orbital elements: semimajor axis $a$, eccentricity $e$, inclination $i$, argument of the pericenter $\omega$, argument of the node $\Omega$, and true anomaly $f$, it yields

$$
\begin{aligned}
\mathcal{H}= & -\frac{\mu}{2 a}-\frac{N}{n} \frac{\mu}{a} \sqrt{1-e^{2}} \cos i \\
& -\frac{N^{2}}{n^{2}} \frac{\mu r^{2}}{8 a^{3}}\left\{2-3 \sin ^{2} i+3 \sin ^{2} i \cos 2 \theta\right.
\end{aligned}
$$




$$
\begin{aligned}
& +\frac{3}{2}\left[2 \sin ^{2} i \cos 2 h+(1-\cos i)^{2} \cos (2 h-2 \theta)\right. \\
& \left.\left.+(1+\cos i)^{2} \cos (2 h+2 \theta)\right]\right\}
\end{aligned}
$$

where the argument of latitude is $\theta=f+\omega$, the argument of the node in the rotating frame is $h=\Omega-N t$, and $n$ is the mean motion of the orbiter.

To apply perturbation theory, we formulate the problem in Delaunay variables $(\ell, g, h, L, G, H)$, where $\ell$ is the mean anomaly, $g$ is the argument of the periapsis, $h$ the argument of the node in the rotating frame, $L=$ $\sqrt{\mu a}$ is the Delaunay action, $G=L \sqrt{1-e^{2}}$ is the modulus of the angular momentum vector, and $H=G \cos i$ is its polar component.

We choose the small parameter of the size of the ratio $N / n$. Therefore, the Coriolis term is a first order effect, and the third body perturbation is at the second order. Then, we apply Lie series method $[20,9,4,38]$ in the style of Deprit, through two steps. First we eliminate the mean anomaly [10] up to the sixth order; then, we remove the node up to the same order.

Thus, up to the sixth order in the small parameter we get the doubly averaged Hamiltonian

$$
\mathcal{K}=-\frac{\mu}{2 a}\left(1+\sum_{m=1}^{6} \frac{\varepsilon^{m}}{m !} \mathcal{K}_{m}\right)
$$

where $\mu$ is set to one in Hill units, $\varepsilon=N / n=a^{3 / 2}=L^{3}$ in Hill units,

$$
\begin{aligned}
\mathcal{K}_{1}= & 2 \eta \cos i, \\
\mathcal{K}_{2}= & \frac{1}{4}\left[\left(2-3 \sin ^{2} i\right)\left(2+3 e^{2}\right)+15 e^{2} \sin ^{2} i \cos 2 g\right], \\
\mathcal{K}_{3}= & \frac{27}{32} \eta \cos i\left[2 \sin ^{2} i+\left(50-17 \sin ^{2} i\right) e^{2}+15 e^{2} \sin ^{2} i \cos 2 g\right], \\
\mathcal{K}_{m}= & (\eta \cos i)^{\operatorname{Mod}(m, 2)} \sum_{j=0}^{m / 2}\left(\sum_{k=0}^{m / 2-j} p_{2 j, 2 k}^{m} e^{2 k}\right)\left(e^{2} \sin ^{2} i\right)^{j} \cos 2 j g, \\
& m=4,5,6
\end{aligned}
$$

the inclination polynomials $p_{j, k}^{i}$ are given on Table 1, where we remark that $\sin ^{2} i=1-H^{2} / G^{2}$, and $\eta=\sqrt{1-e^{2}}=G / L$ is the eccentricity function.

Note that, in addition to the averaged Hamiltonian given by (3), perturbation methods provides explicit transformation equations from averaged to non averaged variables. We obtained full transformation equations up 
Table 1: Inclination polynomials of the averaged Hamiltonian (3).

\begin{tabular}{l}
$p_{0,0}^{4}=-\frac{147}{4}+\frac{531}{16} \sin ^{2} i+\frac{27}{64} \sin ^{4} i$ \\
$p_{0,2}^{4}=\frac{12501}{16}-\frac{25407}{32} \sin ^{2} i+\frac{1647}{8} \sin ^{4} i$ \\
$p_{0,4}^{4}=-\frac{36207}{64}+\frac{51327}{64} \sin ^{2} i-\frac{135189}{512} \sin ^{4} i$ \\
$p_{2,0}^{4}=\frac{8991}{32}-\frac{6615}{32} \sin ^{2} i$ \\
$p_{2,2}^{4}=-\frac{20619}{64}+\frac{36315}{128} \sin ^{2} i$ \\
$p_{4,0}^{4}=-\frac{9855}{512}$ \\
\hline$p_{0,0}^{5}=-\frac{1455}{2}+\frac{71115}{256} \sin ^{2} i+\frac{5265}{1024} \sin ^{4} i$ \\
$p_{0,2}^{5}=\frac{4321275}{256}-\frac{7232565}{512} \sin ^{2} i+\frac{2498445}{1024} \sin ^{4} i$ \\
$p_{0,4}^{5}=-\frac{13089375}{1024}+\frac{18405765}{1024} \sin ^{2} i-\frac{50714505}{8192} \sin ^{4} i$ \\
$p_{2,0}^{5}=\frac{384795}{64}-\frac{2508975}{1024} \sin ^{2} i$ \\
$p_{2,2}^{5}=-\frac{10381815}{1024}+\frac{15246225}{2048} \sin ^{2} i$ \\
$p_{4,0}^{5}=-\frac{1022875}{8192}$ \\
$p_{0,0}^{6}=-14115+\frac{7188135}{512} \sin ^{2} i-\frac{3896265}{2048} \sin ^{4} i+\frac{26055}{2048} \sin ^{6} i$ \\
$p_{0,2}^{6}=\frac{225672585}{512}-\frac{741160455}{1024} \sin ^{2} i+\frac{735822465}{2048} \sin ^{4} i-\frac{98359515}{2048} \sin ^{6} i$ \\
$p_{0,4}^{6}=-\frac{1618613895}{2048}+\frac{53332845}{32} \sin ^{2} i-\frac{18786241005}{16384} \sin ^{4} i+\frac{4170001095}{16384} \sin ^{6} i$ \\
$p_{0,6}^{6}=\frac{893703465}{2048}-\frac{1052086005}{1024} \sin ^{2} i+\frac{13578178635}{16384} \sin ^{4} i-\frac{3660906915}{16384} \sin ^{6} i$ \\
$p_{2,0}^{6}=\frac{132083505}{1024}-\frac{40852035}{256} \sin ^{2} i+\frac{49140675}{1024} \sin ^{4} i$ \\
$p_{2,2}^{6}=-\frac{966987855}{2048}+\frac{3287900085}{4096} \sin ^{2} i-\frac{1258750125}{4096} \sin ^{4} i$ \\
$p_{2,4}^{6}=\frac{847259145}{2048}-\frac{2850049935}{4096} \sin ^{2} i+\frac{2332554975}{8192} \sin ^{4} i$ \\
$p_{4,0}^{6}=-\frac{815947935}{16384}+\frac{865624725}{16384} \sin ^{2} i$ \\
$p_{4,2}^{6}=\frac{1031730345}{16384}-\frac{1032168825}{16384} \sin ^{2} i$ \\
$p_{6,0}^{6}=\frac{13878675}{8192}$ \\
$\frac{1}{6}=1$ \\
\end{tabular}


to the fifth order in the small parameter both in Delaunay and nonsingular elements [11]. General transformation equations of the averaging have thousands of terms, but simplified transformations may be enough for mission design purposes [27].

Hereafter we use the following terminology: When we refer to the firstorder averaging it will be understood that mean elements are used directly as obtained from (3) up to the order $\varepsilon^{2}$. A second order theory refers to the same Hamiltonian plus first order transformation equations. A third order theory, means Eq. (3) truncated up to $\varepsilon^{3}$ plus second order transformation equations. And so on. Thus, the sixth order theory includes all the terms in the Hamiltonian (3) plus fifth order transformation equations.

\subsection{Flow of the averaged problem}

The momenta $L$ and $H$-equivalently $\varepsilon$ and $\sigma=H / L=\sqrt{1-e^{2}} \cos i-$ are integrals of the averaged problem because the conjugate variables $\ell$ and $h$ are cyclic in the averaged Hamiltonian. Therefore, Hamilton equations show that the three-degrees of freedom (averaged) problem is separable: The reduced (i.e., transformed) problem

$$
\frac{\mathrm{d} g}{\mathrm{~d} t}=\frac{\partial \mathcal{K}(g, G ; L, H)}{\partial G}, \quad \frac{\mathrm{d} G}{\mathrm{~d} t}=-\frac{\partial \mathcal{K}(g, G ; L, H)}{\partial g},
$$

involving $g$ and $G$ may be solved first. Then, the rotational motion of $\ell$ and $h$ can be obtained by quadratures

$$
\begin{aligned}
& \ell=\ell_{0}+\int \frac{\partial \mathcal{K}(g(t), G(t) ; L, H)}{\partial L} \mathrm{~d} t, \\
& h=h_{0}+\int \frac{\partial \mathcal{K}(g(t), G(t) ; L, H)}{\partial H} \mathrm{~d} t .
\end{aligned}
$$

Better than finding the general solution of the averaged motion, we are interested in the general description of the flow. More specifically, the integrable reduced system (5) is of one degree of freedom and, therefore, the reduced flow is made of closed curves and equilibria; the latter correspond to frozen orbits of the non averaged model which are of major interest in mission design. Besides, for such perturbed system as the Saturn-Enceladus one, the motion of the node will not be small in general. Then, the knowledge of the frequencies of the motion of $\ell$ and $h$ may help in finding repeat ground-track orbits of the non averaged model, thus easing stability checks.

We note that we do not need the 6th order to give a qualitative description of the dynamics. The lowest order showing only isolated equilibria 
is enough for these purposes. The reduced flow can be studied neglecting constant terms and scaling the time. Therefore, up to the second order, the unique relevant term in the Hamiltonian (3) is $\mathcal{K}_{2}$ which only depends on the parameter $\sigma$. The classical result shows that the so-called Lidov-Kozai resonance determines the flow [35,23]. Thus, values of $\sigma$ with $\sigma^{2}=3 / 5$ divide the flow in three different regions: two with stable circular frozen orbits, with inclinations below $39.2 \mathrm{deg}$ and above $140.8 \mathrm{deg}$ respectively. Circular, frozen orbits are unstable in the other region, but two elliptic, stable, frozen orbits exist with the argument of the periapsis either at 90 or $270 \mathrm{deg}$.

The third order approach requires the term $\mathcal{K}_{3}$ and introduces the second parameter $\varepsilon$. The dynamics of the averaged problem changes and the bounds of the stability regions bend. Bifurcation of elliptic orbits with pericenter at 90 and 270 deg occur along the lines

$$
\varepsilon=\frac{8}{9 \sigma} \frac{3-5 \sigma^{2}}{3+5 \sigma^{2}}
$$

and with pericenter at 0 and $180 \mathrm{deg}$ along the line $\varepsilon=-2 /(9 \sigma)$. Nevertheless, this order shows a degeneracy of equilibria over this last bifurcation line, where orbits with the argument of pericenter at 0 deg and $180 \mathrm{deg}$ are equilibria for any eccentricity. Therefore, it is mandatory to take the fourth order into account for providing the correct qualitative description of the reduced phase space. Thus, elliptic orbits with the argument of the pericenter at 0 deg and 180 do not bifurcate anymore from circular, and elliptic orbits with $g= \pm \pi / 2$ bifurcate from circular at the lines [28]:

$$
\varepsilon=\frac{-36 \sigma\left(3+5 \sigma^{2}\right) \pm 4 \sqrt{5076+1473 \sigma^{2}+4730 \sigma^{4}-27375 \sigma^{6}}}{423+767 \sigma^{2}+1470 \sigma^{4}}
$$

Higher orders in the averaging do not modify the qualitative description of the flow, but introduce quantitative higher order corrections. Thus, up to the sixth order averaging, we find the bifurcation line of circular orbits

$$
\begin{aligned}
0= & 3-5 \sigma^{2}-\frac{9}{8} \sigma\left(3+5 \sigma^{2}\right) \varepsilon-\frac{1}{64}\left(423+767 \sigma^{2}+1470 \sigma^{4}\right) \varepsilon^{2} \\
& -\frac{\sigma}{1024}\left(18563+114578 \sigma^{2}+55755 \sigma^{4}\right) \varepsilon^{3} \\
& -\frac{5}{12288}\left(49334+422433 \sigma^{2}+1033511 \sigma^{4}+436806 \sigma^{6}\right) \varepsilon^{4}
\end{aligned}
$$

that, of course, comprises all the lower order approaches.

Figure 2 shows how the bifurcation lines of circular orbits, where the stability of circular orbits changes, depart from the straight lines $\sigma= \pm \sqrt{3 / 5}$, 


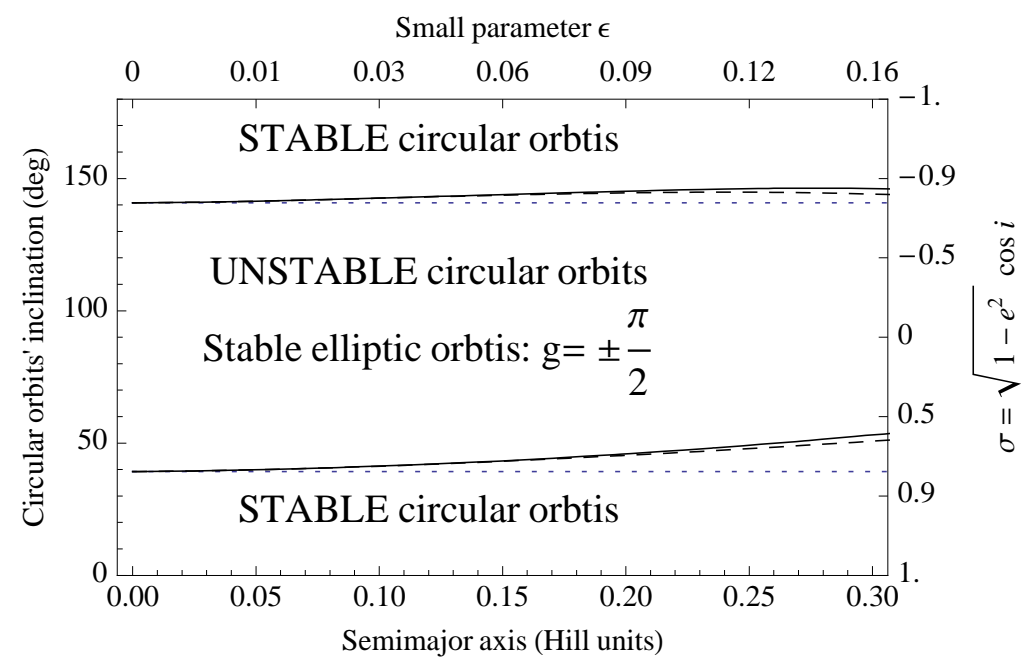

Figure 2: Stability regions for low-eccentricity orbits of Hill problem according to the 2 nd order theory (dotted line), 4th order (dashed), and sixth order (full line).

the gap between different orders increasing with the semimajor axis. Note that this last result is in full agreement with previous numerical results in the literature for non averaged models $[43,31,26]$.

\section{Application to the Saturn-Enceladus system}

We note that Fig. 2 ranges only to $a=0.3$ (or $\varepsilon \sim 0.16$ ). Further than this distance we cannot make general claims about results provided by the analytical theory, because planar direct orbits depart from Keplerian. This semimajor axis roughly matches 1.5 times Enceladus' equatorial radius, cf. Fig. 3, so that even the sixth order analytical theory can hardly describe the general dynamical behavior of an orbiter about Enceladus. However, from observation $[31,43,45]$, we know that high inclination orbits exist that are less deformed at larger distances than the corresponding planar orbits. Therefore, we investigate the degree of agreement between the averaged and non averaged models only for orbits close to the bifurcation line of circular orbits. In addition, we only deal with direct inclination orbits, that, according to the analytical results of this paper and periodic orbits computations in the literature, will provide the higher inclinations for stable almost circular orbits. 


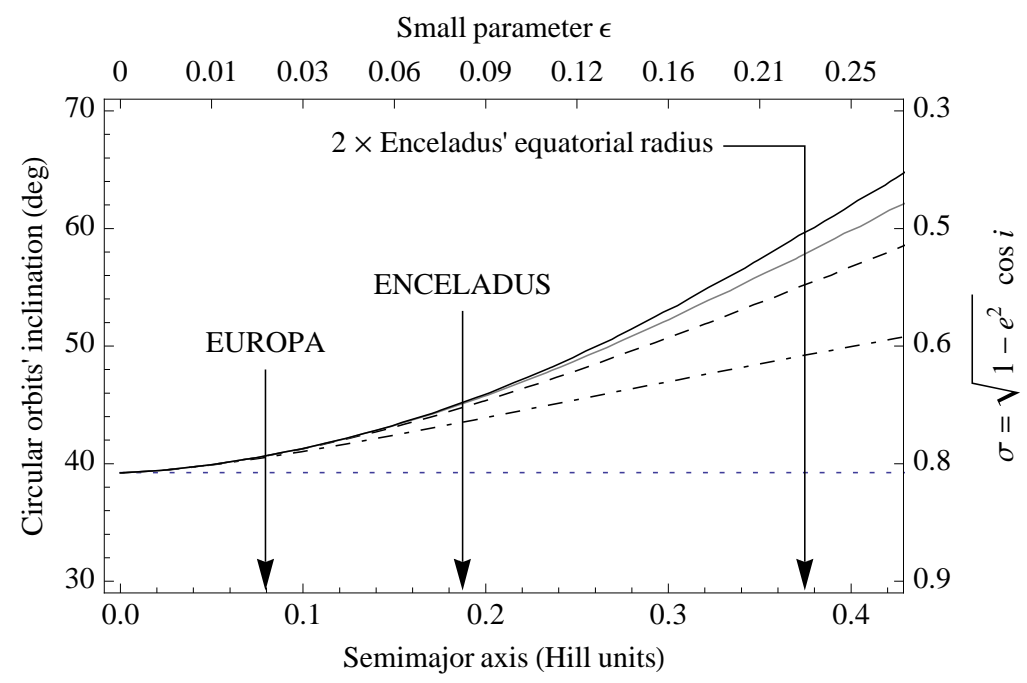

Figure 3: Detail on the region of direct inclination orbits on Fig. 2, showing the equatorial radius of Europa and Enceladus. Bifurcation lines of circular orbits: 2nd, dotted, 3rd, dash-dotted, 4th, dashed, 5th, gray, and 6th order theories, black.

Figure 3 also highlights a semimajor axis two times the equatorial radius of Enceladus (of $256 \mathrm{~km}$ ), a distance that has been recently proposed for the science orbit of a prospective Enceladus mission $[45,5]$. For this semimajor axis, we note that the sixth order averaging predicts stable low-eccentricity orbits below a mean inclination of $\sim 60 \mathrm{deg}$, providing an explanation for the 8:35 repeat cycle, stable, periodic orbit found in [45] with an average inclination of $\sim 61 \mathrm{deg}$.

\subsection{Frozen orbits computation}

Hill's case of close orbits to the smaller primary is a simplification of the restricted three-body problem, which in turn is a simplification of real models. Therefore, the final goal of our theory is not the generation of ephemerides but to help in mission designing for artificial satellite missions about planetary satellites, where frozen orbits are of major interest.

For given values of the parameters $\varepsilon$ and $\sigma$ determined by the mission, a number of frozen orbits may exist. They are computed as equilibria of the 
reduced flow given by (5):

$$
\frac{\partial \mathcal{K}}{\partial G}=0, \quad \frac{\partial \mathcal{K}}{\partial g}=0
$$

To each equilibrium of the doubly reduced phase space corresponds a torus of quasi periodic solutions in the non averaged model, because we are free to chose the initial values of $\ell$ and $h$.

As Delaunay variables are singular for zero eccentricity orbits, for which the argument of the periapsis is not defined, the flow is properly studied using the invariants related to the symmetries of the problem introduced through the averagings [8]. For practical purposes the central body will have a non zero radius and, putting aside rectilinear orbits, the reduced phase space may be studied in the variables introduced by Coffey, Deprit, and Deprit, see $[7,33]$. These variables show that circular orbits are always equilibria. Therefore we find frozen orbits with zero mean eccentricity, either stable or unstable, for any point on the $(\sigma, \varepsilon)$ parameters plane. On the contrary, elliptic orbits are safely studied in Delaunay variables.

We note that the left side equation in (10), which corresponds to the time evolution of $G$, is always factorized by $\sin 2 g$; so it always vanishes in the "principal meridians" $g=0, \pi / 2, \pi, 3 \pi / 2$. Substitution of these values of $g$ in the right side equation in Eq. (10) will result in a polynomial in $\eta$. The real roots such that $|\sigma| \leq \eta \leq 1$, if any, will provide the eccentricities of the elliptic equilibria. Putting aside high eccentricity orbits, we only find stable elliptic frozen orbits with the argument of the pericenter $\pi / 2$ and $3 \pi / 2$, which bifurcate from circular at the bifurcation lines given by (9).

Below we present several examples that justify the effort in computing a 6th order theory to reach the quasi periodicity condition in Hill problem when applied to the Saturn-Enceladus system.

\subsection{Elliptic frozen orbits}

We choose a semimajor axis of $346 \mathrm{~km}$, roughly 1.35 times the equatorial radius of Enceladus of $256 \mathrm{~km}$ or 0.187369 units of Hill problem. This allows non impact orbits up to a maximum eccentricity $e_{m}=0.259$. The sixth order bifurcation line on Fig. 3 shows that stable elliptic frozen orbits with argument of the pericenter $g= \pm \pi / 2$ bifurcate from circular at the inclination $i=50.1 \mathrm{deg},(\sigma=0.64139)$. Therefore, we choose $\sigma=0.635$, close after the bifurcation has occurred. For this value the classical approach predicts a frozen orbit with $e=0.4245$. Despite this eccentricity corresponds to an impact orbit with the surface of Enceladus, we compute initial conditions 
directly from mean elements $(\ell=h=0)$ and perform a long term propagation in the non averaged model. We repeat the long term propagations for the second to the sixth order theories, but undoing the transformation equations in order to obtain osculating elements from the mean ones provided by the averaging. Initial osculating elements in the different cases are given in Table 2, while Fig. 4 shows a comparative of the one year long term evolution in the non averaged model of the osculating eccentricity (left column) and argument of pericenter (right column) for the different theories.

Table 2: Elliptic frozen orbit for $\varepsilon=0.127217, \sigma=0.635(\omega=90 \mathrm{deg}$, $\ell=\Omega=0$ ). The semimajor $a$ appears in Hil units and $i$ in degrees.

\begin{tabular}{lllllll} 
Averaged: & 1st order & 2nd order & 3rd order & 4th order & 5th order & 6th order \\
\hline$a$ & 0.252948 & 0.252948 & 0.249617 & 0.248808 & 0.248294 & 0.247987 \\
$e$ & 0.424522 & 0.424522 & 0.227642 & 0.142806 & 0.106585 & 0.087654 \\
$i$ & 45.4659 & 47.9415 & 49.8033 & 51.0761 & 51.5706 & 51.7691 \\
\hline
\end{tabular}

Thus, concerning Fig. 4, we see that initial conditions computed directly from mean elements obtained with the first-order averaging do not correspond to a frozen orbit: the argument of the pericenter tours continuously over the $360 \mathrm{deg}$, and the eccentricity oscillates between 0.164055 and 0.668469. The second order theory barely improves the orbit, which definitely does not remain frozen at all. The third order theory clearly reduces the excursion in eccentricity, that now remains between 0.0347224 and 0.421042 , but still provides an impact orbit. The fourth order theory reduces the amplitude of the eccentricity oscillations and brings the orbit closer to the frozen condition; the argument of the pericenter remains most of the time either in the vicinity of 90 or $270 \mathrm{deg}$, but the reduced flow remains of the rotational type. One needs to resort to the fifth order theory to find oscillatory motion on the $(e, \omega)$-plane. The orbit remains frozen on average but the instantaneous oscillations in eccentricity range from 0.017452 to 0.258789 , and the argument of the pericenter oscillates $\sim \pm 41 \mathrm{deg}$ about its average value of $90 \mathrm{deg}$. Finally, the sixth order theory improves the frozen orbit condition, with an eccentricity oscillation of \pm 0.09 about the average value $e=0.15$, and the oscillations of the pericenter are reduced to $\pm 27 \mathrm{deg}$.

The evolution in the plane $(e \cos \omega, e \sin \omega)$ is presented in Fig. 5 for the fifth and sixth order theories, to which we superimposed the homoclinic trajectory provided by fifth and sixth analytical theory, respectively, to see the degree of agreement between averaged and non averaged results. 

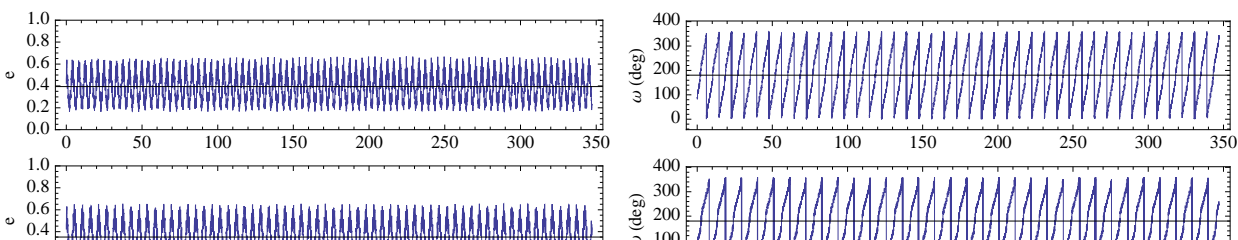

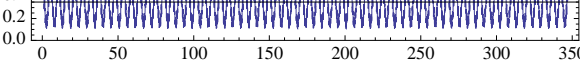
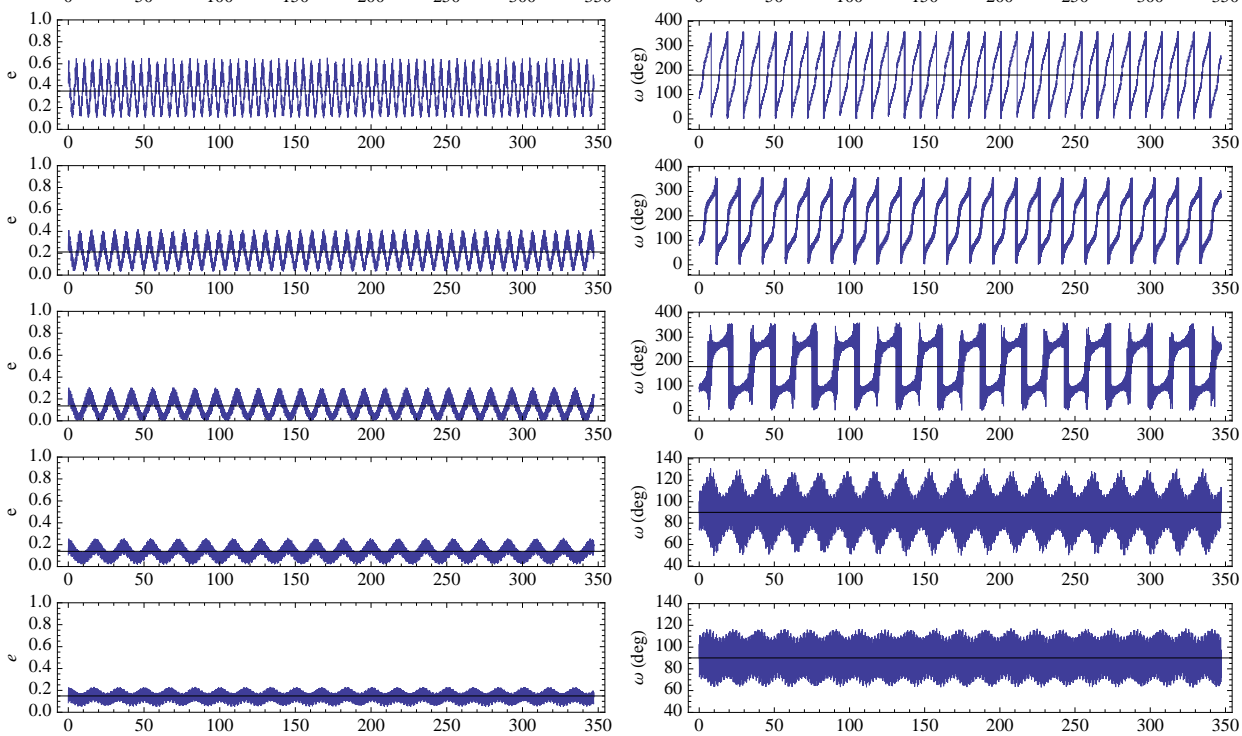

Figure 4: Long term evolution of the osculating eccentricity (left) and argument of the pericenter (right) of the elliptic frozen orbit. From top to bottom: 1st, 2nd, 3rd, 4th, 5th, and 6th order perturbation theory. Abscissas are days. 

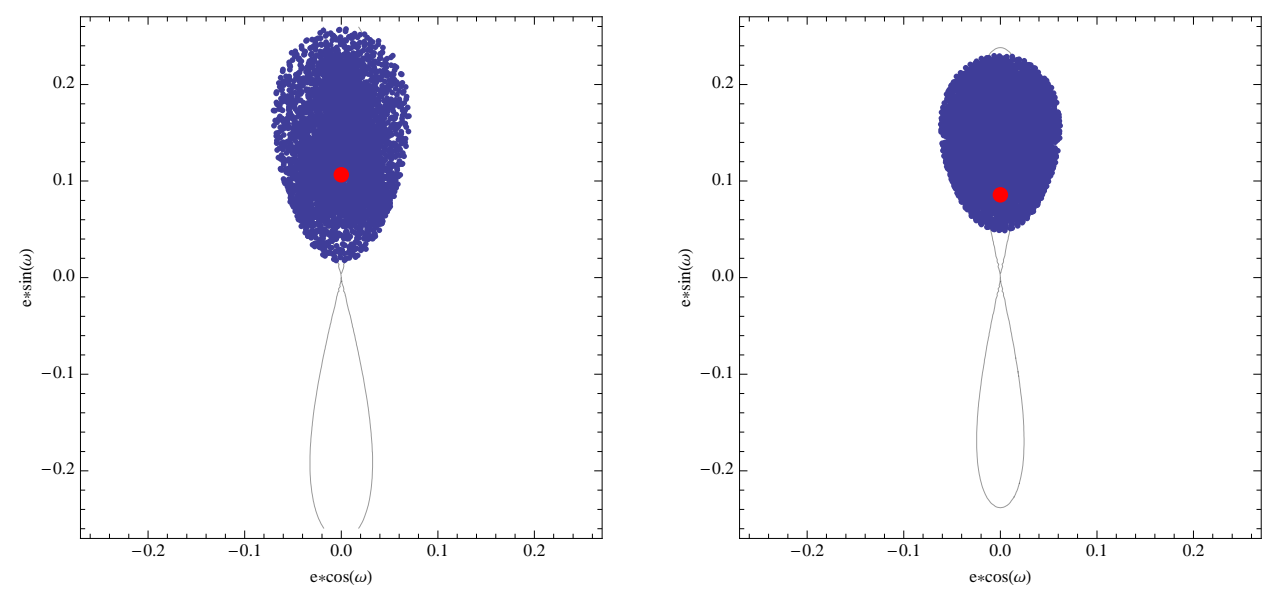

Figure 5: Eccentricity-pericenter evolution. Left: 5th order theory. Right: 6 th order. Red dots mark starting initial conditions as obtained from Table 2 .

\subsection{Circular frozen orbits}

Better results are obtained for the case of circular orbits. For the example above of $\varepsilon=0.127217$ the sixth order averaging predicts almost circular, stable, frozen orbits for inclinations below $i=50.1 \mathrm{deg},(\sigma=0.64139)$. Therefore, we choose $\sigma=0.65$ and, as before, test the reliability of results provided by the different orders of the averaging. Initial osculating elements in the different cases are given in Table 3, while Fig. 6 presents a comparative of the long term evolution in the non averaged model of the osculating eccentricity and argument of the pericenter for the different theories.

Table 3: Circular frozen orbit for $\varepsilon=0.127217, \sigma=0.650(\omega=\Omega=\ell=0)$. Averaging: 2nd order 3rd order 4th order 5th order 6th order

\begin{tabular}{llllll}
$a$ (Hill units) & 0.252948 & 0.259126 & 0.259619 & 0.259771 & 0.259748 \\
$e$ & 0.0000000 & 0.0323685 & 0.0383653 & 0.0408547 & 0.0415872 \\
$i$ (deg) & 51.5048 & 51.4183 & 51.3136 & 51.3216 & 51.3069 \\
\hline
\end{tabular}

Strictly speaking, we cannot claim that we obtain a frozen orbit because the argument of pericenter of our computed orbits always circulates. Nevertheless, we feel satisfied with the analytical description of the dynamics if we are able to reach stable, low eccentricity orbits with small amplitude long period oscillations in the inclination and eccentricity. 

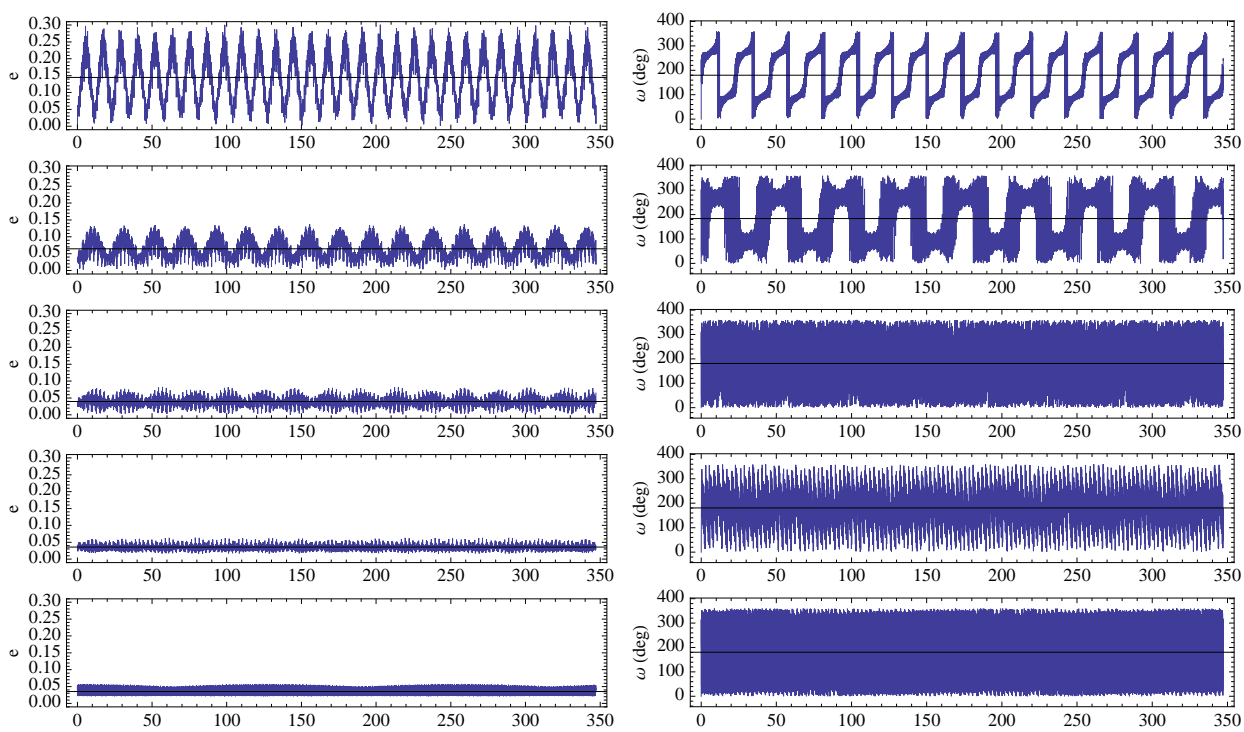

Figure 6: Long term evolution of the osculating eccentricity (left) and argument of the pericenter (right) of the circular frozen orbit. From top to bottom: 2nd, 3rd, 4th, 5th, and 6th order perturbation theory.

The first row of Fig. 6 shows that initial conditions provided by the second order theory do not correspond to a circular frozen orbit. In addition to the medium period oscillations due to the proper dynamics, we find long period oscillations (as high as $\sim 0.3$ in eccentricity) that produce impact orbits. In fact, the higher initial inclination provided by the 2nd order theory of $i=51.5 \mathrm{deg}$, cf. the first column of Table 3, corresponds to the flow after the bifurcation has occurred and the circular orbits change to unstable. From the rigth plot of the first row on Fig. 6, we see that the pericenter long term evolution changes from pointing approximately half of the time to the vicinity of $90 \mathrm{deg}$, and the other half to $270 \mathrm{deg}$.

The 3rd order theory (second row on Fig. 6) provides much better results, and the long period oscillations reduce to 0.137 in eccentricity. However, the initial inclination provided by the analytical theory $i=51.4 \mathrm{deg}$ (cf. the second column of Table 3) still leads to the undesired flow, with unstable circular orbits after the bifurcation happened, and the argument of the pericenter remains for most of the time either in the neighborhood of 90 or 270 deg.

The third row on Fig. 6 shows results provided by the 4 th order theory. Now the initial inclination provided by the theory $i=51.3 \mathrm{deg}$ (third column 
on Table 3) leads to a long term evolution corresponding to the flow before the change to circular orbits instability, and the eccentricity oscillations reduce to $\sim 0.08$. Results corresponding to the fifth and sixth order theories are shown in the last two rows on Fig. 6. The orbit clearly circularizes, with an eccentricity oscillation of \pm 0.024 over the average value of $e=0.0358$ for the 5th order, and an oscillation of \pm 0.018 over the average $e=0.0355$ for the 6 th. Besides, long term effects on inclination are almost negligible when compared with the medium period oscillations.

The evolution on the eccentricity-pericenter diagram is provided on Fig. 7, where we note the improvements produced by the sixth order theory over the acceptable ones of the fifth order theory, producing a more regular behavior with smaller eccentricity oscillations.
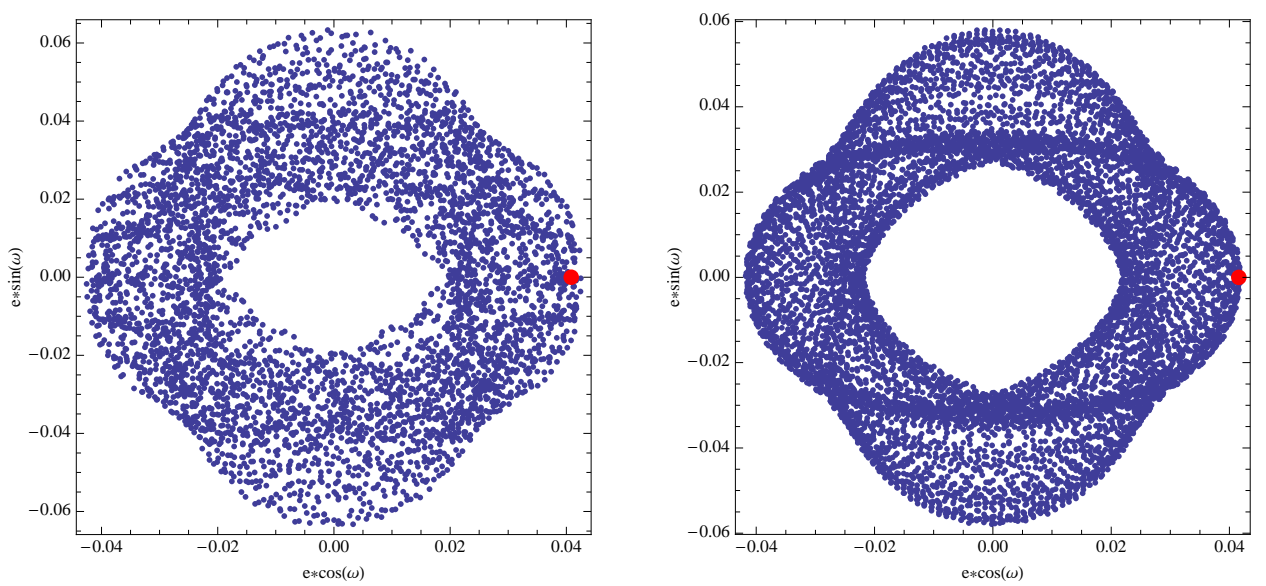

Figure 7: Eccentricity-pericenter evolution. Left: 5th order theory. Right: 6th order. Red dots mark starting initial conditions as obtained from Table 3 .

Alternatively to the temporal analysis above, a frequency analysis using the Fast Fourier Transform (FFT) shows how initial conditions obtained from different orders of the analytical theory are affected by the undesired frequencies that defrost the orbital elements. Thus, Fig. 8 shows the FFT analysis of the instantaneous eccentricity of the almost circular orbit in the example above. The grey line corresponds to initial conditions obtained from the second order analytical theory; the red one to the fourth order analysis, and dots corresponds to the sixth order theory.

We note on Fig. 8 the high amplitude of the low frequencies that appear in the second order approach, which are due to the non frozen condition 


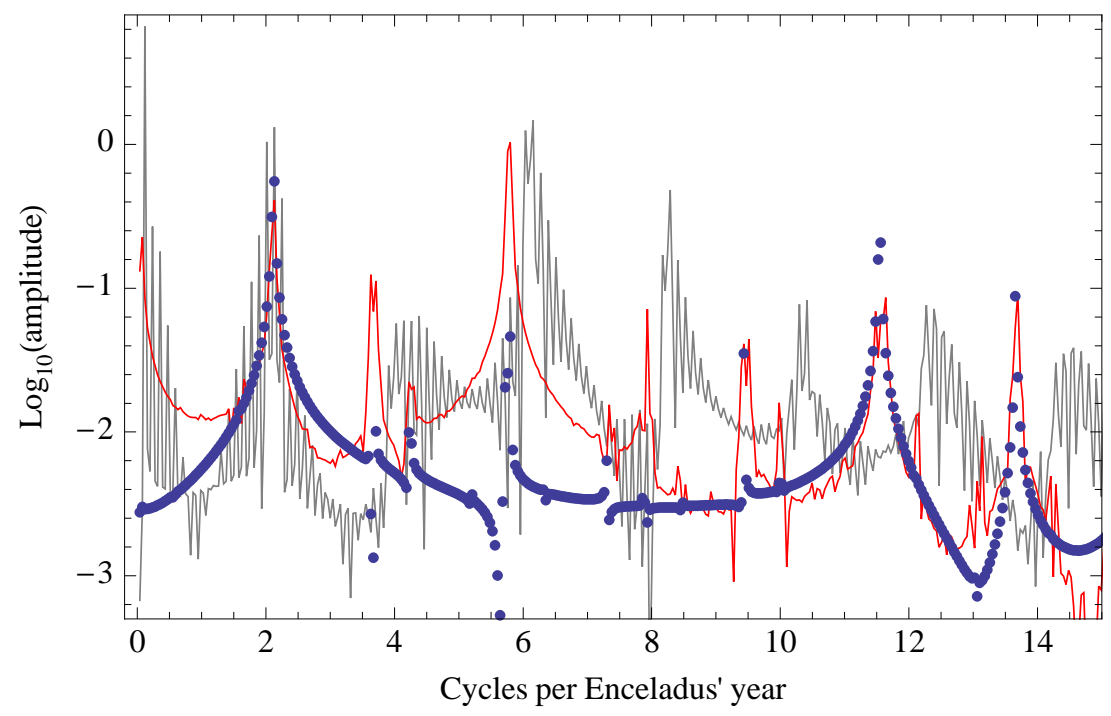

Figure 8: FFT analysis of the instantaneous eccentricity of the low-altitude, almost circular orbit, using the 2nd (gray line), 4th (red), and 6th (dots) order theories.

that produces circulation of the pericenter with non constant eccentricity the complete excursion lasting around eight Enceladus' orbits. The fourth order clearly captures the main frequencies of the motion, although an undesirable low frequency with non negligible amplitude still remains. Finally, while most of the frequencies match, the sixth order notably reduces the amplitude of lower frequencies and makes the long period perturbations almost negligible, thus freezing the orbit. Note that the semiannual effects related to frequencies of about 2 cycles per Enceladus' orbit cannot be eliminated because they are due to the third body dynamics.

\subsection{Enceladus' prospective science orbit}

Finally, we test the reliability of the theory for larger distances to the origin. Thus, for $a_{0}=0.374737$ Hill units $(\varepsilon=0.229399)$, corresponding to two equatorial radius of Enceladus, the sixth order averaging predicts the change to unstable circular orbits at an inclination $i_{0}=63.2472 \mathrm{deg}$, or $\sigma=0.450141$. We choose $\sigma=\cos 60^{\circ}$, and mean elements $a=a_{0}=512.6$ $\mathrm{km}, e=0, i=60 \mathrm{deg}, e \cos \omega=e \sin \omega=0, h=\ell=0$. After undoing the fifth order transformation equations we get $a=560.865 \mathrm{~km}, e=0.165106$, $i=61.5471 \mathrm{deg}, \omega=\Omega=\ell=0$. The propagation of corresponding initial 
conditions shows long term stability, with negligible long period effects in the osculating elements. Figure 9 shows the time evolution of the osculating elements. The semimajor axis averages to $513.5 \mathrm{~km}$ and shows medium period oscillations of $\pm 44.5 \mathrm{~km}$ associated to the mean motion of Enceladus about Saturn. The eccentricity averages to $e=0.1433$ with medium period oscillations of \pm 0.068 , the inclination oscillates $\pm 3.7 \mathrm{deg}$ over its average value of $58.6 \mathrm{deg}$, the pericenter circulates, as well as the node which moves at a rate of $\sim 18 \mathrm{deg} /$ day. Finally, the minimum pericenter altitude oscillates between 133.3 and $232.1 \mathrm{~km}$, with an average value $d=183.65 \mathrm{~km}$. The evolution on the eccentricity-pericenter diagram is provided on Fig. 10.

We note that, for this semimajor axis, the change of circular orbits stability predicted by the fifth order averaging occurs at $57.8 \mathrm{deg}$ of inclination, thus predicting a wrong (unstable) behavior for the $60 \mathrm{deg}$ orbit. Therefore, for distances up to two Enceladus equatorial radius, a sixth order perturbation theory is sufficient to accurately predict the initial conditions of almost circular frozen orbits. For distances closer to Enceladus, a fifth order or less order theory may suffice.

Now we deal with the calcultion of periodic orbits form the twice averaged system. At a first sight, initial conditions of a periodic orbit might be estimated from the mean elements of a frozen orbit by imposing the commensurability between the mean motion of the frozen orbit and its mean node rate in the rotating frame. An obvious theoretical objection to this procedure is that an analytical solution obtained by averaging first the mean anomaly first, and then the argument of the node in the rotating frame, is not valid for resonant frequencies of these two angles, as the trouble of small divisors arises. Therefore, the computation of periodic orbits from averaged solutions needs to be performed to different approaches, see for instance $[21,25$.] Here we use a practical point of view that works well when several averages are executed, see [41] for a similar approach in the context of the elliptic restricted three body problem.

Given a frozen orbit $g=g_{0}, G=G_{0}$,

$$
\frac{\partial \mathcal{K}\left(g_{0}, G_{0}, H, L\right)}{\partial L}=n_{\ell}, \quad \frac{\partial \mathcal{K}\left(g_{0}, G_{0}, H, L\right)}{\partial H}=n_{h},
$$

where $n_{\ell}$ and $n_{h}$ are constant, Eq. (6) gets converted into

$$
\ell=\ell_{0}+n_{\ell} t, \quad h=h_{0}+n_{h} t,
$$

and $n_{\ell}$ and $n_{h}$ result to be the frequencies of the (averaged) motion of $\ell$ and $h$, respectively. Then, by requiring commensurability between the mean 

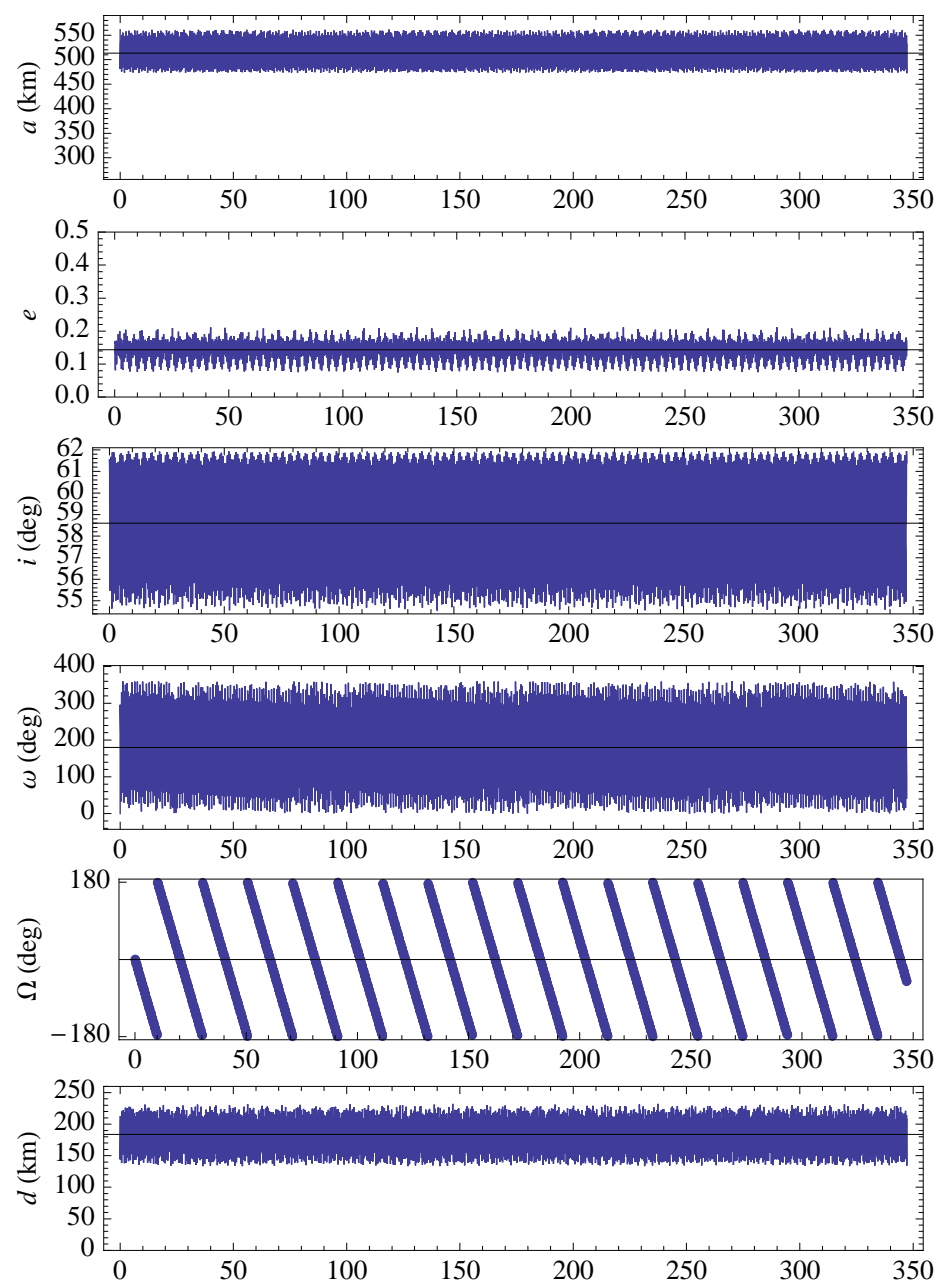

Figure 9: Long-term evolution of the osculating orbital elements and pericenter distance $d$ of the circular frozen orbit with mean $a=512.6 \mathrm{~km}$. 


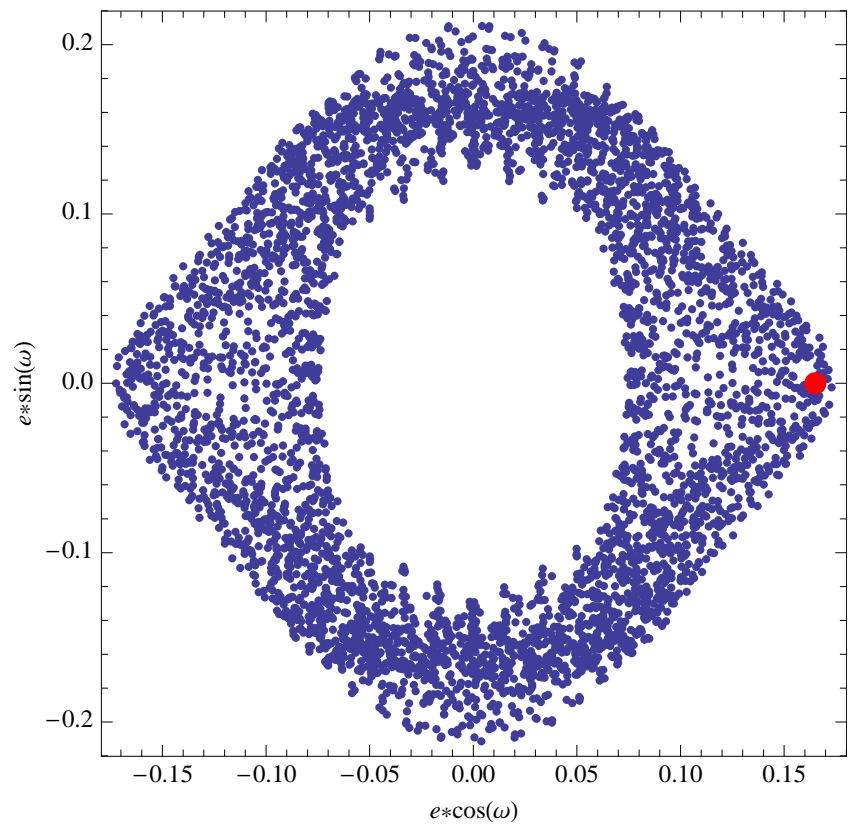

Figure 10: Eccentricity-pericenter evolution. 6th order.

motions of $\ell$ and $h$

$$
P n_{\ell}=D n_{h},
$$

where $P$ and $D$ are integers, we can compute mean elements of an orbit that, up to the order of the perturbation theory, will repeat the ground-track in the non averaged problem.

Thus, for instance, if we require for the Enceladus science mission to be a repeat ground-track orbit we proceed as follows:

- First, we check the ratio $n_{h}$ over $n_{\ell}$ for the mean elements of the Enceladus orbit above ( $a=2 \times$ Enceladus' equatorial radius, $i=60$ deg). We get the value

$$
-0.235862 \approx-\frac{96}{407} \sim-\frac{1}{4}
$$

- Therefore, if we want the mean elements to repeat in a 1:4-cycle, we fix $i=60 \mathrm{deg}$ and recompute $a$ by requiring that $4 n_{h}=n_{\ell}$. This gives $a=532.335 \mathrm{~km}$, about $20 \mathrm{~km}$ larger than our initial approach. We further check from (9) that the new orbit belongs to the stability region for circular orbits, and proceed. 
- Compute the period of the new orbit:

$$
T=\frac{2 \pi}{n_{h}}=4 \times \frac{2 \pi}{n_{\ell}}=112628.8 \mathrm{~s} .
$$

- After undoing the transformation equations of the sixth order theory and propagating corresponding initial condition in the non averaged model, we check that the orbit does not exactly repeat the ground track (see left plot of Fig. 11). This is mostly because we used mean values to compute the repeat condition. However, straightforward differential corrections improve initial conditions until getting the required path.

To further emphasize the limited application of the analytical theory at these long distances, in terms of the Hill radius, we continue the family of 1:4 periodic orbits for variations of the Jacobi constant in the direction of decreasing inclinations. The orbits soon change to increasing instability until its termination on a highly unstable, planar, direct orbit. As presented in the right plot of Fig. 11, the termination orbit looks like a non Keplerian ellipse centered at the origin when shown in the rotating frame.
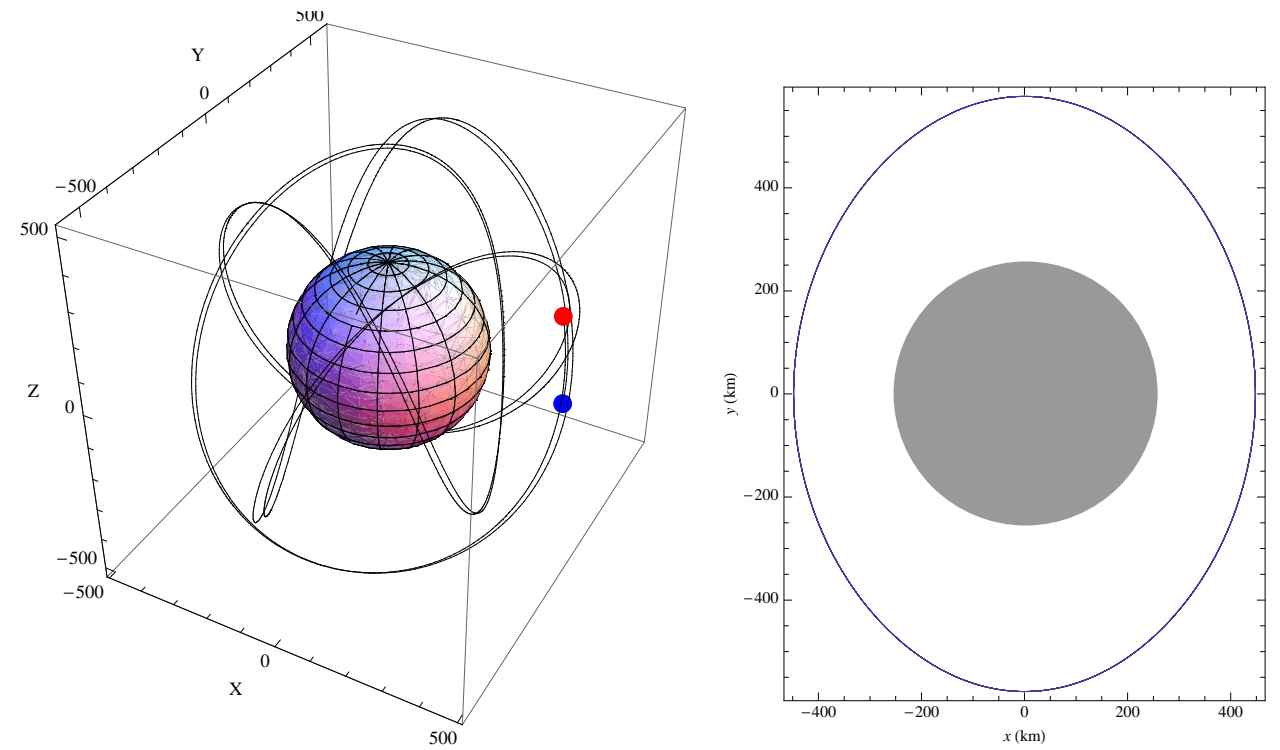

Figure 11: Left: Almost periodic, science orbit after a 1:4-cycle. Right: termination, direct (non Keplerian) orbit of the family of 1:4-repeat groundtrack periodic orbits.

The 1:4 resonance may be not adequate for most science objectives, and longer repeat cycles could be desirable. The steps described above can be 
used for computing higher order resonances of the mean elements that may succeed in finding longer repeat cycles. Thus, for instance, Fig. 12 shows the evolution of the orbital elements of a stable 7:31-repeat ground track orbit, with variations in the osculating elements similar to those of the 8:35 solution proposed in Ref. [45]. The initial conditions in the rotating frame of the 7:31-repeat orbit are $(\mathrm{km}$ and $\mathrm{km} / \mathrm{s})$ :

$$
\begin{aligned}
& x=-343.9027647526488, \\
& y=319.4186962208731, \\
& z=0, \\
& \dot{x}=-0.01705476339728647, \\
& \dot{y}=-0.03667693137743753, \\
& \dot{z}=-0.1117751681934419,
\end{aligned}
$$

and period $T=779346.508048146$ seconds. Besides, we checked that the periodic orbit is stable as predicted by the sixth order averaging.

\section{Ephemerides validation}

In this section we relax the Hill's assumptions and demonstrate robustness of the proposed Enceladus orbits to perturbations associated with a more realistic force model. We perform long term simulations including $n$ body effects from the Sun, Jupiter, Saturn, and the large moons of Saturn (Mimas, Tethys, Dione, Rhea, Titan, and Iapetus). To be consistent with the force model based on average, we ignore non spherical terms at Enceladus; however nonzero even zonal terms $\left(J_{2}, J_{4}, J_{6}\right.$, and $\left.J_{8}\right)$ at Saturn are included because they are important terms in the modeling of the body ephemerides generation. The body locations and orientations are based on publicly available data from the Jet Propulsion Laboratory ${ }^{2}$.

Figures 13 and 14 give the eccentricity vector evolution for three of the preceding example orbits. The initial conditions for the ephemeris runs are the same as those given for the 6th order eccentric, 6th order circular,

\footnotetext{
${ }^{2}$ URL: http://naif.jpl.nasa.gov/naif/spiceconcept.html (cited 1 Jan 2009).

URL: ftp://naif.jpl.nasa.gov/pub/naif/generic_kernels/spk/planets/a_old_versions/ de405.bsp (cited 1 Jan 2009).

URL: ftp://naif.jpl.nasa.gov/pub/naif/generic_kernels/spk/satellites/a_old_versions/ sat242.bsp (cited 1 Jan 2009).

URL: ftp://naif.jpl.nasa.gov/pub/naif/generic_kernels/pck/pck00008.tpc (cited 1 Jan 2009).
} 

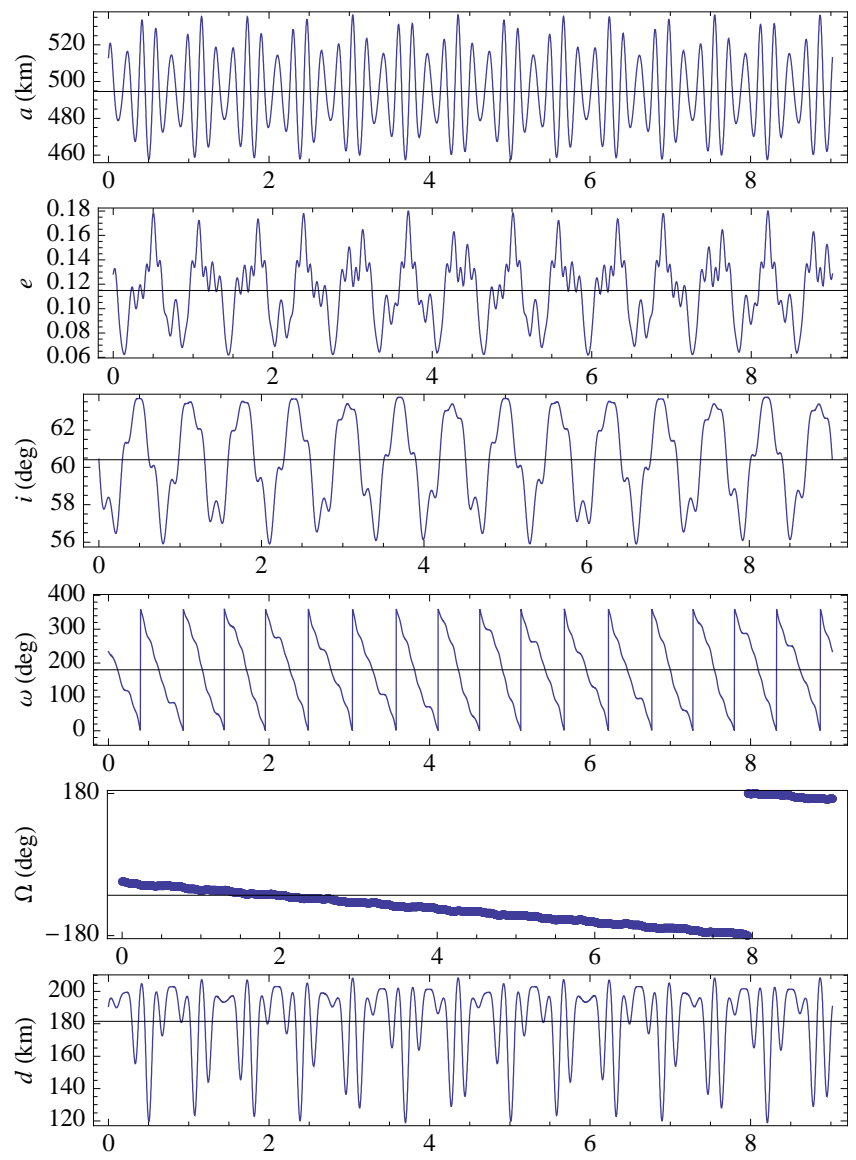

Figure 12: Evolution of the osculating orbital elements and pericenter distance $d$ of the $7 / 31$ repeat ground-track, stable, frozen orbit.

and high inclination prospective science orbit. The epoch for the results we are showing is arbitrarily chosen as Jan. 1, 2028 (Julian Date $=2461772.0$ ). A quick comparison of Figs. 13 and 14 to Fig. 5 (right side), Fig. 7 (right side), and Fig. 10 respectively show very good qualitative agreement. Several different epochs were evaluated and all exhibited similar results.

We note that inclusion of the non spherical terms at Enceladus does have an appreciable effect on the evolution of the eccentricity vector of closer orbits, in many cases changing the behavior from a predicted circulation to a libration and vice versa. To properly account for the expected large $J_{2}$ and $C_{2,2}$ terms at Enceladus, the formal averaging model should be adjusted. 

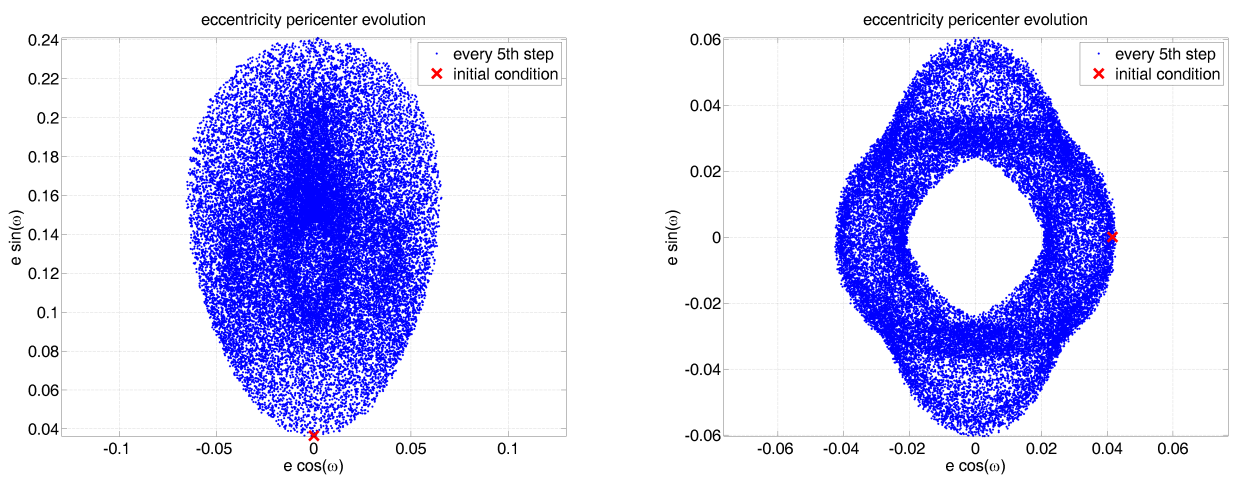

Figure 13: One year ephemeris propagation starting at 6th order elliptic frozen orbit from Table 2 (left plot) and 6th order circular frozen orbit from Table 3 (right plot).

\section{Conclusions}

For the motion of an orbiter in a planetary satellite system, the radius of validity of averaging theories is usually constrained to small distances of the orbiter to the natural satellite. This radius of validity is related to the ratio of satellite's mean motion to orbiter's, a small parameter that scales the third body perturbation and suggests the use of perturbation theory to extend the validity of the averaged dynamics to larger distances to the origin.

However, how much useful averaging can be for describing the long term dynamics of perturbed two body problems, its validity is obviously limited to the set of trajectories that look like conics. Therefore, one cannot naively expect that reaching higher and higher orders in a perturbation theory will eventually provide mean elements useful to assess possible orbiter motion in a global context.

That is exactly the case of the Saturn-Enceladus system, where the shape of direct, equatorial orbits about Enceladus depart from Keplerian ellipses as close to Enceladus as one and a half of its radius. Regrettably enough, proposed science missions about Enceladus may require larger distances to make possible global coverage with the moderate inclinations required to find orbit stability under the huge pull of Saturn. Nevertheless, even in the extreme case of Enceladus, the averaged dynamics may be used to describe the long term behavior of high inclination orbits in a region that extends to two Enceladus' radius, despite it requires at least a sixth order averaging. 


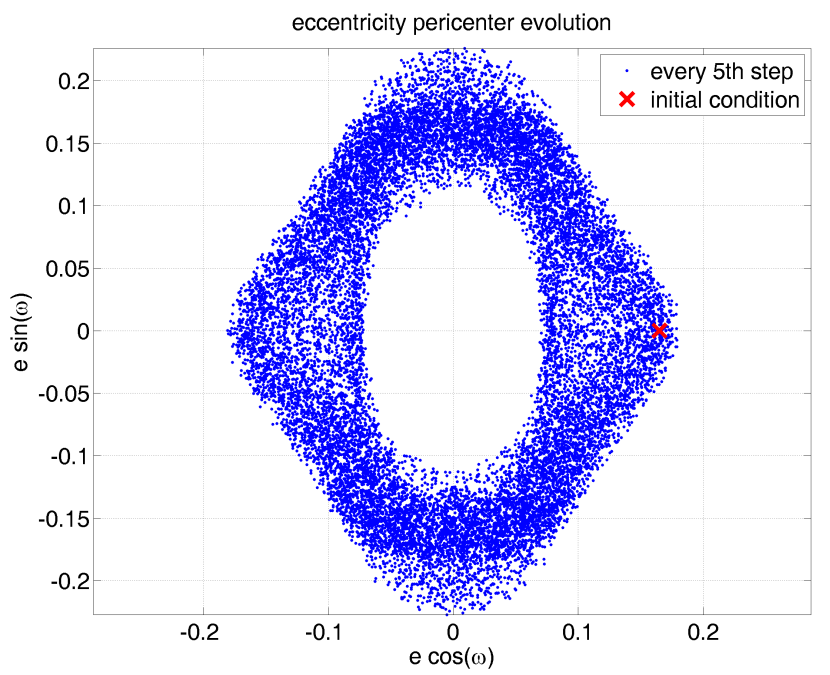

Figure 14: One year ephemeris propagation starting at the orbit from Fig. 10 .

Thus, high inclination stable orbits about Enceladus predicted by the sixth order averaging are demonstrated to show long term stability in the non averaged model. Furthermore, a selected set of stable solutions is checked to survive in the transition from the simplified dynamics to an ephemeris model.

In this study we ignore the non sphericity of Enceladus, an assumption that is somewhat justified for the semimajor axis of proposed science orbits ( two times Enceladus' equatorial radius), for which the third body perturbation clearly dominates the dynamics. A correct representation of the dynamics closer to Enceladus requires the inclusion of at least the oblateness and non sphericity coefficients of the central body. Because Enceladus is tidally locked to Saturn, this does not cause any problem from the point of view of perturbation theory, except for the large increase in the size of the formal expression to handle.

\section{Appendix: On the validity of the averaging}

Independently of the previous knowledge of the almost Keplerian or non Keplerian character of the orbits of Hill problem, we can estimate the validity of the averaging from the bifurcation line of circular orbits as follows. 


\subsection{An estimate by Newton-Raphson method}

The accuracy of the bifurcation line of circular orbits increases with the order of the theory. Thus, (9) is accurate up to the order of $\varepsilon^{4}$. However, the contributions of terms of the order of $\varepsilon^{5}$ may be soon important, constraining the validity of the theory to small values of $\varepsilon$. One way of checking this fact is to solve (9) for $\sigma$ up to the order of our theory. Starting from $\sigma_{0}= \pm \sqrt{3 / 5}$, three iterations are enough for a Newton-Raphson procedure to converge to

$$
\sigma= \pm \sqrt{\frac{3}{5}}-\frac{27}{40} \varepsilon \mp \frac{325}{128} \sqrt{\frac{3}{5}} \varepsilon^{2}-\frac{26897}{6400} \varepsilon^{3} \mp \frac{11118173}{1474560} \sqrt{\frac{3}{5}} \varepsilon^{4}+\mathcal{O}\left(\varepsilon^{5}\right),
$$

that is accurate up to the order of $\varepsilon^{4}$, and must agree to the same order with (9). Note that, while a series convergence criteria show that the series in the right side of (14) might converge in the ball with Hill radius, its convergence may be poor for relatively small values of $\varepsilon$.

Departure of both Eqs. (9) and (14) from the real (unknown) bifurcation line means that the contribution of terms of the order of $\varepsilon^{5}$ is not negligible. This applies also to Eqs. (9) and (14) themselves, which will depart from each other roughly at the same point. Therefore, a graphic representation of both solutions, Eqs. (9) and (14), will reveal the point where the two lines separate from each other, thus manifesting the $\varepsilon$ value from which higher order contributions in $\varepsilon$, missed in the analytical theory, are important. Figure 15 shows this graphic estimator, where departure of both curves from each other at $\varepsilon \approx 0.16$, or $a \approx 0.3$ Hill units, is clearly observed -in very nice agreement with previous considerations.

\subsection{An estimate by the reconstruction of periodic orbits}

Another way of checking how accurate the bifurcation line of circular orbits provided by the analytical theory is when far from the origin, is by comparison of that line with a similar bifurcation line constructed from a dense set of points where almost circular, periodic orbits of the non averaged problem change their stability character (see Ref. [32]).

We make use of the mean frequencies commensurability procedure described above to compute a variety of almost circular, stable, resonant, periodic orbits close to the bifurcation line (9) provided by the analytical theory. For each periodic orbit we continue the corresponding family until detecting the change to instability, and compute average elements of the bifurcation periodic orbit. When projected on the (averaged) $a-i$ plane, each bifurcation orbit is represented by a point. Figure 16 shows these points from a variety 


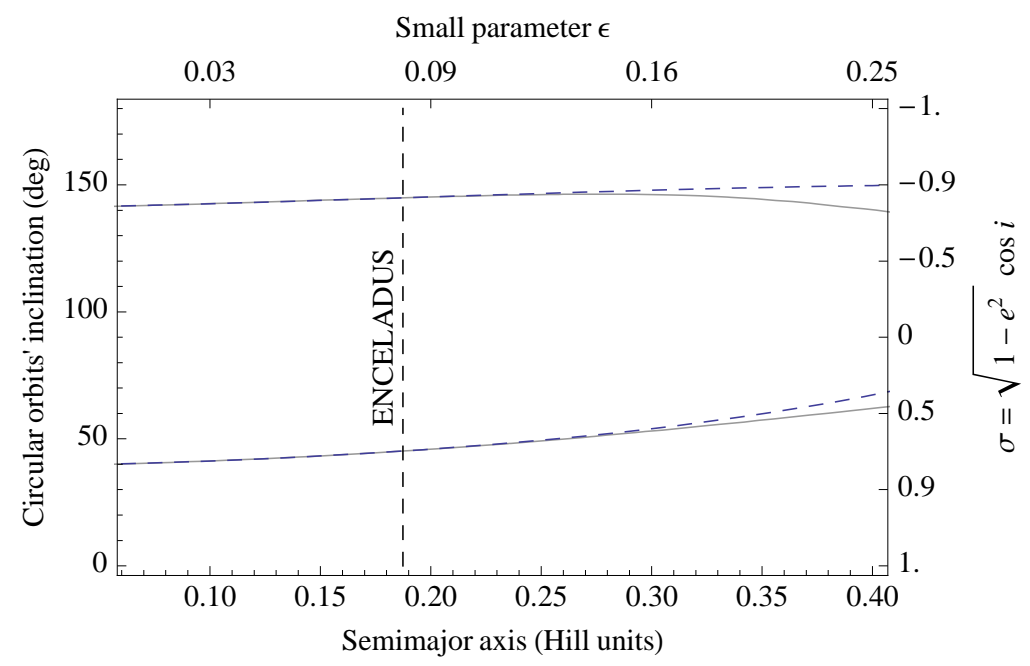

Figure 15: Analytic bifurcation line (full) vs its Newton-Raphson approximation (dashed).

of resonances jointly with the bifurcation line of (9). Again, the departure from each other of both bifurcation lines seems to support previous estimations. Besides, we note that the branch of the bifurcation line of circular orbits corresponding to direct inclination orbits, sticks to the periodic orbits' bifurcation line up to larger values of the small parameter (longer distances) than in the retrograde case, also in accordance with computations. Finally, we note that the fact that the Newton-Raphson approximation given by (14) remains closer to the periodic orbit's bifurcation line than the solution (9), for both direct and retrograde branches, must be considered just a lucky chance.

\section{Acknowledgements}

We acknowledge support from Projects ESP 2007-64068 (M.L.) and MTM 2005-08595 (J.F.P.) of the Government of Spain. This research has made use of NASA's Astrophysics Data System.

\section{References}

[1] Aiello, J., "Numerical Investigation of Mapping Orbits about Jupiter's Icy Moons," paper AAS 2005-377, Aug 2005 


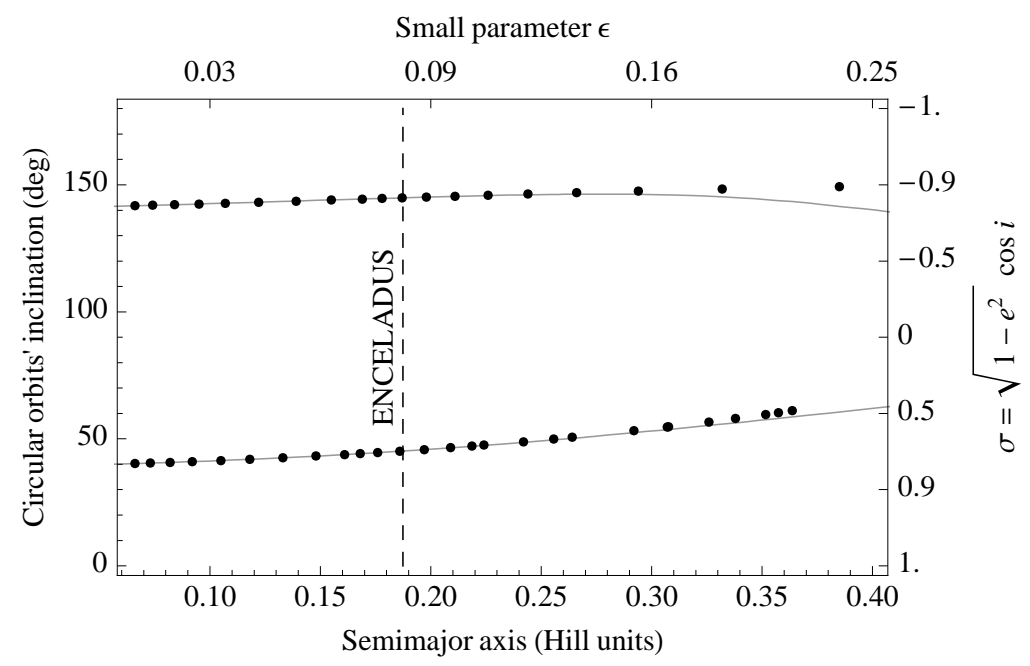

Figure 16: Analytic bifurcation line (full line) vs periodic orbit computation (dots).

[2] Broucke, R.A., "Stability of Periodic Orbits in the Elliptic Restricted Three-Body Problem," AIAA Journal, Vol. 7, No. 6, 1969, pp. 10031009

[3] Broucke, R.A., "Long-Term Third-Body Effects Via Double Averaging," Journal of Guidance, Control, and Dynamics, Vol. 26, No. 1, 2003, pp. 27-32

[4] Campbell, J.A., Jefferys, W.H. "Equivalence of the Perturbation Theories of Hori and Deprit," Celestial Mechanics, Vol. 2, No. 4, 1970, pp. $467-473$

[5] Casotto, S., Padovani, S., Russell, R.P., Lara, M., "Detecting a Subsurface Ocean from Periodic Orbits at Enceladus," AGU 2008 Fall Meeting, 15-19 Dec 2008, San Francisco, CA.

[6] Coffey, S., Deprit, A., "Third-Order Solution to the Main Problem in Satellite Theory," Journal of Guidance, Control, and Dynamics, Vol. 5, No. 4, 1982, pp. 366-371

[7] Coffey, S., Deprit, A., and Deprit, E., "Frozen Orbits for Satellites Close to an Earth-like Planet," Celestial Mechanics and Dynamical Astronomy, Vol. 59, No. 1, 1994, pp. 37-72 
[8] Cushman, R., "Reduction, Brouwer's Hamiltonian and the Critical Inclination." Celestial Mechanics, Vol. 31, Dec. 1983, pp. 409-429; errata: Vol. 33, Aug. 1984, p. 297

[9] Deprit, A., "Canonical Transformations Depending on a Small Parameter," Celestial Mechanics, Vol. 1, No. 1, 1969, pp. 12-30

[10] Deprit, A., "Delaunay Normalisations," Celestial Mechanics, Vol. 26, No. 1, 1982, pp. 9-21

[11] Deprit, A., Rom, A., "The Main Problem of Artificial Satellite Theory for Small and Moderate Eccentricities," Celestial Mechanics, Vol. 2, No. 2, 1970, pp. 166-206

[12] Folta, D., Quinn, D., "Lunar Frozen Orbits," Paper AIAA 2006-6749, Aug 2006

[13] Gómez, G., Marcote, M., Mondelo, J.M., "The Invariant Manifold Structure of the Spatial Hill's Problem," Dynamical Systems: An International Journal, Vol. 20, No. 1, 2005, pp. 115-147

[14] Hamilton, D.P., Krivov, A.V., "Dynamics of Distant Moons of Asteroids," Icarus, Vol. 128, No. 1, 1997, pp. 241-249

[15] Hénon, M., "Exploration Numérique du Problème Restreint. II. Masses Égales, Stabilité des Orbites Périodiques," Annales d'Astrophysique, Vol. 28, No. 2, 1965, pp. 992-1007

[16] Hénon, M., "Numerical Exploration of the Restricted Problem. V. Hills Case: Periodic Orbits and Their Stability," Astronomy and Astrophysics, Vol. 1, 1969, pp. 223-238

[17] Hénon, M., "Numerical Exploration of the Restricted Problem. VI. Hill's Case: Non-periodic Orbits," Astronomy and Astrophysics, Vol. 9, 1970, pp. 24-36

[18] Hénon, M., "Vertical Stability of Periodic Orbits in the Restricted Problem II. Hills Case," Astronomy 65 Astrophysics, Vol. 30, 1974, pp. 317321

[19] Hill, G.W., "Researches in the Lunar Theory," American Journal of Mathematics, Vol. I, 1878, pp. 129-147 
[20] Hori, G.-I, "Theory of General Perturbations with Unspecified Canonical Variables," Publications of the Astronomical ociety of Japan, Vol. 18, No. 4, 1966, pp. 287-296

[21] Jefferys, W.H., "A New Class of Periodic Solutions of the Threedimensional Restricted Problem," The Astronomical Journal, Vol. 71, No. 2, 1966, pp. 99-102

[22] Kovalevsky, J., "Sur la Théorie du Mouvement dun Satellite à Fortes Inclinaison et Excentricité, in The Theory of Orbits in the Solar System and in Stellar Systems, Proceedings from Symposium no. 25 held in Thessaloniki, 1964. Edited by G.I. Kontopoulos. IAU Symp. No. 25. Thesssaloniki, Academic Press, London, 1966, pp. 326-344

[23] Kozai, Y., "Secular Perturbations of Asteroids with High Inclination and Eccentricity," The Astronomical Journal, Vol. 67, No. 9, 1962, pp. $591-598$

[24] Kozai, Y., "Motion of a Lunar Orbiter," Publications of the Astronomical Society of Japan, Vol. 15, No. 3, 1963, pp. 301-312

[25] Kozai, Y., "Stationary and Periodic Solutions for Restricted Problem of Three Bodies in Three-dimensional Space," Publications of the Astronomical Society of Japan, Vol. 21, No. 3, 1969, pp. 267-287

[26] Lam, T., Whiffen, G.J., "Exploration of Distant Retrograde Orbits around Europa," Paper AAS05-110, Jan 2005

[27] Lara, M., "Simplified Equations for Computing Science Orbits Around Planetary Satellites," Journal of Guidance, Control and Dynamics, Vol. 31, No. 1, 2008, pp. 172-181

[28] Lara, M., Palacián, J.F., "Hill Problem Analytical Theory to the Order Four: Application to the Computation of Frozen Orbits Around Planetary Satellites," Mathematical Problems in Engineering, Vol. 2009, 2009., 753653, 18 pages.

[29] Lara, M., Palacián, J.F., Russell, R.P., "Averaging and Mission Design: The Paradigm of and Enceladus Orbiter," Paper AAS09-199, Feb 2009 20 pages.

[30] Lara, M., Russell R.P., "On the Family $g$ of the Restricted Three-body Problem". Monografías de la Real Academia de Ciencias de Zaragoza, Vol. 30, 2007, pp. 51-66 
[31] Lara, M., Russell, R.P., Villac, V., "Classification of the Distant Stability Regions at Europa," Journal of Guidance, Control, and Dynamics, Vol. 30, No. 2, 2007, pp. 409-418

[32] Lara, M., San-Juan, J.F., "Dynamic Behavior of an Orbiter Around Europa," Journal of Guidance, Control and Dynamics, Vol. 28, No. 2, 2005, pp. 291-297

[33] Lara, M., San-Juan, J.F., and Ferrer, S., "Secular Motion around Triaxial, Synchronously Orbiting, Planetary Satellites: Application to Europa," Chaos: An Interdisciplinary Journal of Nonlinear Science, Vol. 15, No. 4, 2005, pp. 1-13

[34] Lara, M., Scheeres, D.J., "Stability Bounds for Three-dimensional Motion Close to Asteroids," The Journal of the Astronautical Sciences, Vol. 50, No. 4, 2002, pp. 389-409

[35] Lidov, M.L., "The Evolution of Orbits of Artificial Satellites of Planets under the Action of Gravitational Perturbations of External Bodies," Planetary and Space Science, Vol. 9, No. 10, 1962, pp. 719-759. Translated from Iskusstvennye Sputniki Zemli, No. 8, 1961, pp. 5-45

[36] Lidov, M.L., Yarskaya, M.V., "Integrable Cases in the Problem of the Evolution of a Satellite Orbit under the Joint Effect of an Outside Body and of the Noncentrality of the Planetary Field," Kosmicheskie Issledovaniya, Vol. 12, Mar-Apr 1974, pp. 155-170

[37] Lo, M.W., Williams, B.G., Bollman, W.E., Han, D., Hahn, Y., Bell, J.L., Hirst, E.A., Corwin, R.A., Hong, P.E., Howell, K.C., "Genesis Mission Design," Paper AIAA-1998-4468, Aug 1998

[38] Mersman, W.A., "A New Algorithm for the Lie Transformation," Celestial Mechanics, Vol. 3, No. 1, 1970, pp. 81-89

[39] Orlov, A.A., "Second-Order Short-Period Solar Perturbations in the Motion of the Satellites of Planets, Bulletin of the Institute of Theoretical Astronomy, Vol. 43, No. 12, 1970, pp. 302-309

[40] Palacián, J.F., "Dynamics of a Satellite Orbiting a Planet with an Inhomogeneous Gravitational Field," Celestial Mechanics and Dynamical Astronomy, Vol. 98, No. 4, 2007, pp. 219-249 
[41] Palacián, J.F., Yanguas, P., Fernández, S., Nicotra, M.A., "Searching for Periodic Orbits of the Spatial Elliptic Restricted Three-body Problem by Double Averaging ," Physica D, Vol. 213, No. 1, 2006, pp. 15-24

[42] Paskowitz, M.E., Scheeres, D.J., "Orbit Mechanics about Planetary Satellites Including Higher Order Gravity Fields," paper AAS 2005190, presented at the 2005 Space Flight Mechanics Meeting, Copper Mountain, Colorado, Jan 2005

[43] Russell, R.P., "Global Search for Planar and Three-dimensional Periodic Orbits Near Europa," Journal of the Astronautical Sciences, Vol. 54, No. 2, 2006, pp. 199-226

[44] Russell, R.P., Brinckerhoff, A.T., "Circulating Eccentric Orbits around Planetary Moons," Journal of Guidance, Control, and Dynamics, Vol. 32, No. 2, 2009, pp. 423-435

[45] Russell, R.P., Lara, M., "On the Design of an Enceladus Science Orbit," Acta Astronautica, Vol. 65, No. 1-2, 2009, pp. 27-39

[46] San-Juan, J.F., Lara, M., "Normalizaciones de Orden Alto en el Problema de Hill," Monografías de la Real Academia de Ciencias de Zaragoza, Vol. 28, 2006, pp. 23-32

[47] Scheeres, D.J., Guman, M.D., Villac, B.F., "Stability Analysis of Planetary Satellite Orbiters: Application to the Europa Orbiter," Journal of Guidance, Control, and Dynamics, Vol. 24, No. 4, 2001, pp. 778-787

[48] Szebehely, V., Theory of Orbits - The Restricted Problem of Three Bodies, Academic Press, New York, 1967

[49] Vashkov'yak, M.A., "A Numerical-Analytical Method for Studying the Orbital Evolution of Distant Planetary Satellites, Astronomy Letters, Vol. 31, No. 1, 2005, pp. 64-72.

[50] Vashkov'yak, M.A., Teslenko N.M. "Refined Model for the Evolution of Distant Satellite Orbits, Astronomy Letters, Vol. 35, No. 12, 2009, pp. $850-865$

[51] Villac, B., Lara, M., "Stability Maps, Global Dynamics and Transfers," AAS Paper 05-378, Aug 2005 